\title{
Maintenance of Normal Stress Tolerance in the Moss Physcomitrella patens Lacking Chloroplastic CuZn-Superoxide Dismutase
}

\author{
Yuya Higashi' ${ }^{1}$, Katsuaki Takechi' ${ }^{1}$, Hiroyoshi Takano', Susumu Takio, ${ }^{1,2}$ \\ ${ }^{1}$ Graduate School of Science and Technology, Kumamoto University, Kumamoto, Japan \\ ${ }^{2}$ Center for Marine Environment Studies, Kumamoto University, Kumamoto, Japan \\ Email: stakio@gpo.kumamoto-u.ac.jp
}

Received 4 February 2015; accepted 10 March 2015; published 12 March 2015

Copyright (C 2015 by authors and Scientific Research Publishing Inc.

This work is licensed under the Creative Commons Attribution International License (CC BY). http://creativecommons.org/licenses/by/4.0/

\section{(c) (i) Open Access}

\begin{abstract}
Superoxide dismutases (SODs) catalyze the dismutation of superoxide and play an important role in reducing oxidative stress in plants. Based on in-gel SOD activity staining, chloroplasts of the moss Physcomitrella patens have two CuZn-SODs as the major SOD isozymes and minor SODs, including a Fe-SOD and two Mn-SODs. To investigate the contribution of chloroplastic SODs to stress tolerance in $P$. patens, we generated a double mutant lacking chloroplastic CuZn-SOD genes. The mutant did not show any differences in comparison to the wild type based on the growth of protonemata on normal and high-salt media, extractable activities of the other SODs after culture on normal and high-salt media, and inhibition of $F_{\mathrm{v}} / F_{\mathrm{m}}$ under stress conditions (high-salt, high-light, and high-temperature). These results indicate that chloroplastic CuZn-SODs do not play a principal role in oxidative stress tolerance in chloroplasts under the investigated conditions. These findings explain the previously reported unusual response of $P$. patens to copper deficiency, in which chloroplastic CuZn-SODs are preferentially inactivated but cytosolic CuZn-SODs are unaffected.
\end{abstract}

\section{Keywords}

Chloroplast, Knockout Mutant, Physcomitrella patens, Stress Tolerance, Superoxide Dismutase

\section{Introduction}

Superoxide dismutase (SOD) catalyzes the dismutation reaction of superoxide radical ( $\mathrm{O}_{2}^{--}$) to hydrogen peroxide $\left(\mathrm{H}_{2} \mathrm{O}_{2}\right)$ and molecular oxygen $\left(\mathrm{O}_{2}\right)$ and plays an important role in protecting cell against oxidative damage caused by reactive oxygen species (ROS) [1]. In plants, SOD enzymes are classified into three isotypes by their

How to cite this paper: Higashi, Y., Takechi, K., Takano, H. and Takio, S. (2015) Maintenance of Normal Stress Tolerance in the Moss Physcomitrella patens Lacking Chloroplastic CuZn-Superoxide Dismutase. American Journal of Plant Sciences, 6, 591-601. http://dx.doi.org/10.4236/ajps.2015.65064 
metal cofactors, namely, copper-zinc SOD (CuZn-SOD), iron SOD (Fe-SOD) and manganese SOD (Mn-SOD) [2]. Since phospholipid membranes are impermeable to $\mathrm{O}_{2}^{--}$, multiple SODs are present to remove $\mathrm{O}_{2}^{--}$in different compartments within the plant cells [3]. Among intracellular compartments, chloroplasts are at particular risk of oxygen toxicity, because molecular $\mathrm{O}_{2}$ can be photoreduced to $\mathrm{O}_{2}^{\cdot-}$ by electrons from photosystem I (PSI) [4]. Chloroplasts of land plants express major CuZn-SOD and minor Fe-SOD. Therefore, chloroplastic CuZn-SOD is thought to play an important role in preventing the oxidative damage associated with photosynthesis.

The moss Physcomitrella patens is used as model plant due to the high frequency of homologous recombination [5] and availability of the whole genome information [6]. Frank et al. [7] reported high tolerance of $P$. patens to salt, drought and osmotic stresses. Since chloroplasts are subjected to oxidative stresses under these stress conditions, it was expected that the moss chloroplasts would have a high stress scavenging system. Recently, we analyzed SOD isozymes of $P$. patens and found several unusual properties in the expression of chloroplastic SOD isozymes [8]. From the genome database, four CuZn-SOD genes (two cytosolic and two chloroplastic isoforms), two Fe-SOD genes (extracellular and chloroplastic isoforms), and two Mn-SOD genes (a previously identified mitochondria isoform and a newly identified but unexpressed isoform) were predicted [8]. Based on SOD activity staining, isolated chloroplasts showed activity of CuZn-SOD but not other SOD isozymes, suggesting that CuZn-SODs are key SOD isozymes for oxidative stress response in chloroplasts [8]. Under copper deficient conditions, chloroplast CuZn-SOD isozymes were preferentially inhibited, while the activities of other SOD isozymes were not affected. In the moss Barbula unguiculata [9] [10] and seed plants Arabidopsis [11] and tobacco [12], Fe-SOD activities were promoted by copper deficiency and a copper responsible cis-element was detected in the promoter region of several Fe-SOD genes [10]. Induction of Fe-SOD activity during copper deficiency is explained as the complementary regulation between different isozymes in chloroplastic stress responses under copper deficient conditions [13]. In contrast to these examples, copper deficiency in $P$. patens did not induce Fe-SOD activity and led to an abrupt reduction of the chloroplast SOD level. Therefore, it was expected that the $P$. patens chloroplasts are sensitive to various stressors under copper deficient conditions [8]. In our preliminary experiments, $P$. patens protonemata grown on copper-deficient medium showed the similar tolerance to high-light stress. These results were unexpected as chloroplastic CuZn-SODs are believed to play an important role in oxidative stress tolerance due to their function as $\mathrm{O}_{2}^{--}$scavenger in the water-water cycle [14]. In $P$. patens, there are several possibilities as to why chloroplasts with reduced level of CuZn-SOD are able to maintain stress tolerance. After repeated subcultures of $P$. patens protonemata under copper-deficient conditions, trace amounts of CuZn-SOD activity were detected [8]. Therefore, it is possible that a small amount of CuZn-SOD is able to maintain normal function in the chloroplast. Another possibility is that trace amounts of Fe-SOD or the other SOD isozymes are present, but at the levels below detection. To address the physiological role of chloroplastic CuZn-SODs against stress tolerance in $P$. patens, we generated single- and double-mutants of chloroplastic CuZn-SOD genes (PpCSD1 and PpCSD2). The data show that stress tolerance in chloroplasts was maintained after complete loss of chloroplastic CuZn-SOD.

\section{Materials and Methods}

\subsection{Plant Material, Growth Conditions and Stress Treatments}

Protonemata of P. patens (Gransden Wood strain) were cultured at $25^{\circ} \mathrm{C}$ under continuous light in a BCDAT agar medium [15]. For vegetative propagation, the plants were ground with a homogenizer in sterile water and soaked in the BCDAT agar medium overlaid with a layer of cellophane.

For the high-salt treatment, protonemata were transferred to liquid BCDAT medium containing $0.5 \mathrm{M} \mathrm{NaCl}$ and shaken at $25^{\circ} \mathrm{C}$ under $80 \mu \mathrm{mol} \mathrm{m} \mathrm{m}^{-2}$. For the high-light treatment, protonemata were transferred to liquid BCDAT medium and exposed to a halogen lamp at $25^{\circ} \mathrm{C}$. For the heat treatment, protonemata were transferred to liquid BCDAT medium and shaken at $44^{\circ} \mathrm{C}$ under $80 \mu \mathrm{mol} \mathrm{m} \mathrm{m}^{-2} \mathrm{~s}^{-1}$. For the determination of protonemata growth under high-salt conditions, protonemata were transferred to BCDAT agar medium supplemented with $\mathrm{NaCl}(0,0.1,0.2,0.3$, and $0.4 \mathrm{M})$ and cultured for 8 days.

\subsection{Chloroplast Isolation}

Chloroplasts were isolated according to the method of Hofmann and Theg [16]. The procedure is somewhat 
modified as described below. Protonemata were pre-cultured in the liquid BCDAT medium under continuous illumination $\left(80 \mu \mathrm{mol} \mathrm{m}^{-2} \mathrm{~s}^{-1}\right)$ with sterile air bubbling. Protoplasts were prepared by treatment with driselase (Kyowa Hakko Bio Co., Ltd.) in 8\% mannitol. Protoplasts suspended in the grinding buffer containing $50 \mathrm{mM}$ HEPES-KOH (pH 7.5), $0.33 \mathrm{M}$ sorbitol, $1 \mathrm{mM} \mathrm{MgCl} 2,2 \mathrm{mM} \mathrm{Na}_{2}$ EDTA and $0.1 \%$ (w/v) BSA were ruptured by forcing and the suspension was loaded onto Percoll gradient consisting of $30 \%$ and $80 \%$ steps in grinding buffer. Chloroplasts were recovered from the interface and washed twice with the storage buffer containing $50 \mathrm{mM}$

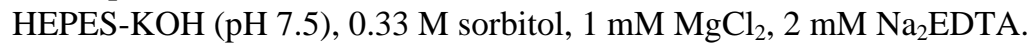

\subsection{Assay for SOD Activity}

Crude extracts were prepared from protonemata (0.2-g fresh weight) by homogenizing in a buffer containing 1 mM EDTA, 1 mM dithiothreitol and $20 \mathrm{mM}$ Tris- $\mathrm{HCl}$ (pH 7.8) in the presence of $20 \mathrm{mg}$ of polyvinylpolypyrrolidone. Protein was quantified spectrophotometrically as described by Bradford [17] with bovine serum albumin as the standard. Native-PAGE was performed at $4^{\circ} \mathrm{C}$ on a $12 \%$ polyacrylamide gel using Laemmli's system in the absence of SDS [18]. The gel was then stained for SOD activity using the riboflavin/nitro blue tetrazolium method [19].

\subsection{Generation of the Knockout Lines}

The knockout construct of PpCSD1 gene was generated as follows. The 5' region (1544 bp) and the 3' region (2614 bp) of PpCSD1 gene were amplified by PCR using genomic DNA as a template. The primer pairs used are indicated in Table 1. The amplified 5' fragment was blunted and cloned into the blunted SphI site of the plasmid pTN3 [15]. The amplified 3' fragment was digested with HindIII and cloned into the HindIII site of the plasmid. The construct was linearized with SalI and the subsequent transformation of $P$. patens was performed as described by Nishiyama et al. [15].

The knockout construct of PpCSD2 gene was generated as follows. The 5' region (2910 bp) and the 3' region (1291 bp) of PpCSD2 gene were amplified by PCR using genomic DNA as a template. The primer pairs used are listed in Table 1. The amplified 5' fragment was digested with HincII and cloned into the blunted SalI site of the plasmid p35S-Zeo (generous gift of Hiwatashi, Y.). The amplified 3' fragment was blunted and cloned into the $X b a \mathrm{I}$ site of the plasmid. The construct was linearized with SacI and the subsequent transformation of $P . p a-$ tens was performed.

\subsection{Genomic Southern Blot Analysis}

Total genomic DNA was isolated as described by Murray and Thompson [20]. Ten micrograms of genomic DNA were digested with DraI (40 U). The digested DNA fragments were fractionated on a $0.8 \%(\mathrm{w} / \mathrm{v})$ agarose gel in TAE buffer and transferred to nylon membrane (Biodyne B, PALL, USA). For hybridization, digoxigenin

Table 1. Primers used for the construction of knockout (KO) plasmids and Southern blot probes in Figure 1. "F" and "R" in primer names indicate forward and reverse, respectively.

\begin{tabular}{|c|c|}
\hline Description of experiment & Primer sequences (from 5’ to 3’) \\
\hline \multirow{2}{*}{ PpCSD1-5' region of $\mathrm{KO}$ construct } & F: GCCTGCGCCTTCCACTTCTAGA \\
\hline & R: CAGTAGACATGCAGCCATTGGT \\
\hline \multirow{2}{*}{ PpCSD1-3' region of $\mathrm{KO}$ construct } & F: CAGGAAATATCATTGCAGGGAGC \\
\hline & R: TGCTTCAACATCCACCTCTAATG \\
\hline \multirow{2}{*}{ PpCSD2-5’ region of $\mathrm{KO}$ construct } & F: CCCTATATCTAAACCAACGCCAAT \\
\hline & R: GGGAGTCAAGCCAGTGATCTT \\
\hline \multirow{2}{*}{ PpCSD2-3’ region of $\mathrm{KO}$ construct } & F: GTGGGTCTAACACCTCTGTAA \\
\hline & R: GGGAGAATGTTAAATAGACCC \\
\hline \multirow{2}{*}{ PpCSD1 Southern blot probe } & F: CTCACTATTGTAGCAGCCACC \\
\hline & R: CAGTAGACATGCAGCCATTGGT \\
\hline \multirow{2}{*}{ PpCSD2 Southern blot probe } & F: GTGGGTCTAACACCTCTGTAA \\
\hline & R: GGGAGAATGTTAAATAGACCC \\
\hline
\end{tabular}


(DIG)-labeled DNA probes complementary to the PpCSD1 region (728 bp) and PpCSD2 region (1291 bp) were synthesized using a PCR Probe Synthesis Kit (Roche Diagnostics, Mannheim, Germany). Prehybridization and hybridization were performed as described previously [21]. The hybridized probes were immune-detected with an alkaline phosphatase-conjugated anti-DIG antibody and visualized using CSPD chemiluminescence substrate as per the supplier's instructions (Roche Diagnostics).

\subsection{RT-PCR Analysis}

Total RNA was isolated with the Sepasol RNA I Super kit (Nacalai Tesque). For RT-PCR cDNA was synthesized from $1 \mu \mathrm{g}$ of total RNA at $42^{\circ} \mathrm{C}$ for 30 min with $1 \mu$ Oligo (dT) primer from the RT-PCR kit (Takara Bio). The primer pairs used for PCR are listed in Table 2. Amplification conditions consisted of an initial denaturation at $94^{\circ} \mathrm{C}$ for $5 \mathrm{~min}$ followed by 30 cycles at $94^{\circ} \mathrm{C}$ for $1 \mathrm{~min}, 55^{\circ} \mathrm{C}$ for $1 \mathrm{~min}$ and $72^{\circ} \mathrm{C}$ for $1 \mathrm{~min}$.

\subsection{Chlorophyll Fluorescence Parameters}

Chlorophyll fluorescence was measured with the FMS1 fluorescence monitoring system (Hansatech, King's Lynn, UK). Before taking the $F_{\mathrm{v}} / F_{\mathrm{m}}$ and NPQ measurements, protonemata were dark-adapted for $30 \mathrm{~min}$. The parameters used were $F_{\mathrm{v}} / F_{\mathrm{m}}$ (maximum quantum efficiency of PSII), NPQ $=\left(F_{\mathrm{m}}-F_{\mathrm{m}}{ }^{\prime}\right) / F_{\mathrm{m}}{ }^{\prime}$ (non-photochemical quenching).

\section{Results}

\subsection{Generation of $\operatorname{csd} 1$, $\operatorname{csd} 2$ Single-Mutants and $\operatorname{csd} 1 / 2$ Double-Mutant}

To investigate the contribution of chloroplastic CuZn-SOD to chloroplast stress tolerance, single mutants lacking the PpCSD1 or PpCSD2 gene (csd1 mutant or csd2 mutant, respectively) and a double mutant (csd1/2 mutant) were isolated by homologous recombination (Figure 1). The knockout construction of PpCSD1 gene contained the nptII gene driven by the CaMV 35S promoter and terminator, flanked by 1544 and 2614 bp of the 5' and 3' end of gene, respectively (Figure 1(a)). The knockout construction of PpCSD2 gene contained the Zeocine gene driven by the CaMV 35S promoter and terminator, flanked by 2910 and 1291 bp of the 5' and 3' end of gene, respectively (Figure 1(b)). The knockout construction of the PpCSD1 gene was introduced into the csd2 single-mutant, and the csd1/2 double-mutant was isolated. To confirm the homologous recombination event in the PpCSD1 or PpCSD2 gene in three mutants (csd1, csd2, and $c s d 1 / 2$ ), Southern blot analysis was performed (Figure $1(\mathrm{c})$ ). Sizes of the hybridized bands were $2.3 \mathrm{kbp}$ for the $c s d 1$ and $1.7 \mathrm{kbp}$ for $c s d 2$, respectively. The band sizes were consistent with what was predicted for homologous recombination of the PpCSD1 and PpCSD2 genes. In the csd1/2 double mutant, the 2.3-kb band was also consistent with the size predicted for homologous recombination at the wild-type PpCSD1 locus.

\subsection{SOD Transcript Level and SOD Activity in Chloroplastic CuZn-SOD Mutants}

To confirm the disruption of genes, accumulation of SOD transcripts in the single mutants and double mutant was analyzed by RT-PCR (Figure 2(a)). The old version (ver. 3.0) of P. patens database included eight SOD isozyme genes. Using this sequence information, we reported that the genes for two chloroplastic CuZn-SODs (PpCSD1, PpCSD2), two cytosolic CuZn-SODs (PpCSD3, PpCSD4), two Fe-SODs (PpFSD1, PpFSD2) and one Mn-SOD (PpMSD1) were expressed and one Fe-SOD gene (PpFSD3), presumably a pseudogene, was not expressed under normal conditions [8]. As shown in Figure 2(a), all SOD genes, except PpFSD3, are expressed in the wild-type under normal conditions. The PpCSD1 and PpCSD2 transcripts were not detected in $c s d 1$ and $c s d 2$, respectively, while the expression of the other SOD genes was not changed in both mutants. In the $c s d 1 / 2$ double-mutant, both the PpCSD1 and PpCSD2 transcripts were not detected, while transcript levels of the other SOD genes were not affected.

To examine SOD activity in the mutants, activity staining for SOD on gels was performed (Figure 2(b)). SOD isozymes are generally classified according to their sensitivity to inhibitors, $\mathrm{KCN}$ and $\mathrm{H}_{2} \mathrm{O}_{2}$ [22]. Fe-SOD was resistant to KCN, but sensitive to $\mathrm{H}_{2} \mathrm{O}_{2}$. Mn-SOD was KCN and $\mathrm{H}_{2} \mathrm{O}_{2}$ resistant, but CuZn-SOD was sensitive to both inhibitors. In our previous report [8], based on their response to inhibitors, three CuZn-SOD bands and three Mn-SODs were identified from protonemata extracts and only the CuZn-SOD band was detected in 
Table 2. Primers used for RT-PCR in Figure 2(a) and Figure 6. "F” and "R" in primer names indicate forward and reverse, respectively.

\begin{tabular}{|c|c|c|c|}
\hline Genes & Primer sequences (from 5' to 3') & Genes & Primer sequences (from 5' to 3') \\
\hline \multirow{2}{*}{ PpCSD1 } & F: AGCGACCACCATGTCCA & \multirow{2}{*}{ РpAРX2 } & F: ACTGGTGGGGCAAATGGTT \\
\hline & R: CTCGACGACACCATCTATC & & R: CTGAGAGCACCTTTCGGTTT \\
\hline \multirow{2}{*}{ PpCSD2 } & F: TATCAACCACATGCCTGCTT & \multirow{2}{*}{ РрАРХЗ } & F: TTCACTGGCAAGCTCCTTCT \\
\hline & R: TTCGTGGATGACGAACG & & R: CAGTTTCCCTGCTTTCTTGG \\
\hline \multirow{2}{*}{ PpCSD3 } & F: ATGTCAACTGGTCCTCATTTCA & \multirow{2}{*}{ РpAРX4 } & F: TTGCGGCTCTATCTCAACCT \\
\hline & R: GTTGAGGCCTGAAGACCGATTA & & R: GCCAAGTCTAACGGGAATCA \\
\hline \multirow{2}{*}{ PpCSD4 } & F: ATGGCTCCTCTGAAGGCTATC & \multirow{2}{*}{ Pp2CP1 } & F: AAGAGTTTCGGTGCTCGTGT \\
\hline & R: TCAAGCTGAAGCCTGAAATC & & R: TCGGGCTTCATGGTCTTT \\
\hline \multirow{2}{*}{ PpFSD1 } & F: ATGGCGACCAGCAGCTTAG & \multirow{2}{*}{ Pp2CP2 } & F: CTGCTACCAATGCACCCACT \\
\hline & R: GGCTCCCCGTAATTGACAAA & & R: ACGATGGGATAATGCAGGTC \\
\hline \multirow{2}{*}{ PpFSD2 } & F: CTTCGCTAGCTCGTGCTTCA & \multirow{2}{*}{ PpPrxQ1 } & F: ATGGCGACTTCAGCTTCCT \\
\hline & R: GACTGGTTCCCCTATGTTCA & & R: GTGCTTTTCTGGCTCAAACTG \\
\hline \multirow{2}{*}{ PpFSD3 } & F: GCAGTTCCATTGCCTTTGAG & \multirow{2}{*}{ PpPrxQ2 } & F: CTCGGAAACGGGCTAAATG \\
\hline & R: TCCCATGTTTCGCACATCTT & & R: AGAGCGCCGAAGAAATCAG \\
\hline \multirow{2}{*}{ PpMSD1 } & F: GCAGCAGCACTCATGCAA & \multirow{2}{*}{ PpPrxQ3 } & F: GACTGTGACCGCTTCTTCTCT \\
\hline & R: AAAGCGCTGAGCCACATC & & R: ATACCACCTTGCCGTTCTTG \\
\hline \multirow{2}{*}{ Actin } & F: TGGTGAGCACAGATTCAACAGA & \multirow{2}{*}{ PpPrxII1 } & F: TGCAAGCAAGGTCTCAGCA \\
\hline & R: CACACCGATCTAGAGCTACAAACA & & R: CACCCACCTCCAAGTTCAAT \\
\hline \multirow{2}{*}{ РpAPX1 } & F: CTTCCTCAGTGCTGGGGTTA & \multirow{2}{*}{ PpPrxII2 } & F: ATCCCTCGCTGTTGTTGCT \\
\hline & R: CCTGGCCCGTTCTTAGTGTA & & R: TGTCCGTCAAGTCCACAGTT \\
\hline
\end{tabular}

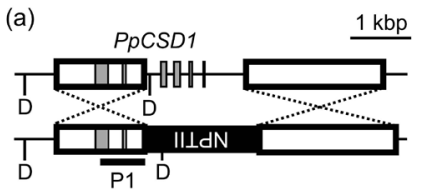

(b)

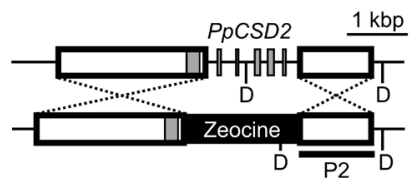

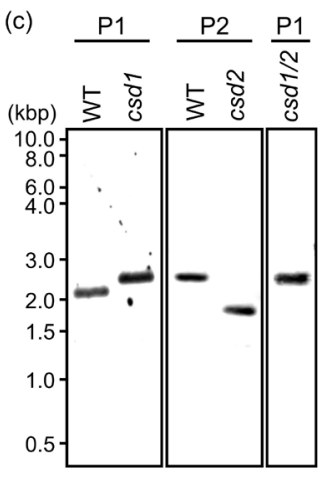

Figure 1. Generation of knockout mutants and confirmation of gene disruption. (a) Scheme of the PpCSD1 genomic region in the wild-type and PpCSD1-knockout mutant. Regions used for homologous recombination are indicated by a box of bold line. The probe used for Southern blotting of the PpCSD1 gene is indicated by a bold bar (P1). Gray box, exon of the PpCSD1 gene; black box, nptII cassette; D, restriction site of DraI; (b) Scheme of the PpCSD2 genomic region in the wild-type and PpCSD2-knockout mutant. Regions used for homologous recombination are indicated by a box of bold line. Probe used for Southern blotting of the PpCSD2 gene is indicated by a bold bar (P2). Gray box, exon of the PpCSD2 gene; black box, zeocine cassette; D, restriction site of DraI; (c) Southern blot analysis of wild-type and csd1, csd2 single-mutants and $c s d 1 / 2$ double-mutant. Genomic DNA $(10 \mu \mathrm{g})$ was digested with DraI and separated on $0.8 \%$ agarose gels. The gels were blotted and hybridized with the probes (P1 or P2) indicated in (a) and (b).

isolated chloroplasts. In this study, the same SOD bands were detected in the wild-type protonemata extract (Figure 2(b), WT). Although chloroplastic CuZn-SOD activity was detected in a single band in the wild-type, the results in the mutants revealed that the single band in the wild-type consisted of two chloroplastic CuZn-SODs bands (Figure 2(b), csd1 and csd2). The csd1/2 double-mutant lacked chloroplastic CuZn-SOD band (Figure 2(b), csd1/2). The other SOD activities did not change in the three mutants. These results suggest that the double mutant lacks chloroplastic CuZn-SOD activity and that any SOD activity cannot complement the lack of chloroplastic CuZn-SODs in the double mutant. 

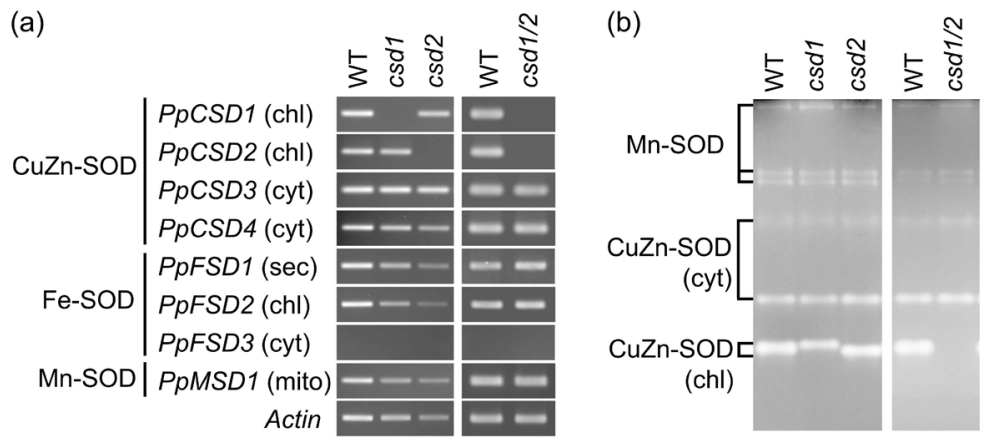

Figure 2. Transcript levels of SOD genes and SOD activity in the chloroplastic CuZn-SOD single and double mutants. (a) Transcript levels of SOD genes in the wild-type, chloroplastic CuZn-SOD single mutants and double mutant. Total RNA was extracted from $P$. patens protonemata cultured on BCDAT solid medium for $6 \mathrm{~d}$ and subjected to RT-PCR. chl, chloroplastic; cyt, cytosolic; sec, secretory; mito, mitochondrial; (b) SOD activity staining of protonemata in the wild-type, chloroplastic CuZn-SOD single-mutants and double-mutant. Crude extracts were prepared from $P$. patens protonemata cultured on BCDAT solid medium for $6 \mathrm{~d}$. The extracts containing $20 \mu \mathrm{g}$ proteins were electrophoresed on a native polyacrylamide gel and stained for SOD activity. cyt, cytosolic; chl, chloroplastic.

\subsection{Identification of Chloroplastic SOD Isozymes in P. patens}

To identify SOD isozyme activity in chloroplasts in P. patens, protoplasts and chloroplasts were prepared from protonemata of the wild-type and $c s d 1 / 2$ double-mutant and their extracts were subjected to native-PAGE (Figure 3). In our previous report, a faint SOD band with resistant to KCN, but sensitive to $\mathrm{H}_{2} \mathrm{O}_{2}$, was present, but it was not detected in isolated chloroplasts [8]. In this study, increased amounts of chloroplast proteins were used for analysis. In the wild-type chloroplasts, the CuZn-SOD band was detected as the major SOD, but minor bands of a Fe-SOD and two Mn-SODs were also detected. The detection of Fe-SOD in chloroplasts of the wild-type and csd1/2 mutant is consistent with the facts that PpFSD2 (chloroplastic Fe-SOD gene) was expressed in both the wild-type and $c s d 1 / 2$ mutant (Figure 3). It was unexpected that two Mn-SOD activity bands were detected in chloroplasts, because only single Mn-SOD gene was deposited on the old version of the $P$. patens data base. Based on the new version of the P. patens database (COSMOSS Ver 3.1), two Mn-SOD genes (PpMSD1, PpMSD2) are predicted to be present in the genome with three transcript variants of PpMSD1. The three variants of PpMSD1 were expressed in both the wild-type and csd1/2 mutant, while PpMSD2 was not amplified using genomic DNA and cDNA (data not shown). Therefore, the three Mn-SOD bands in the in-gel SOD staining appear to be derived from the three variants of PPMSD1. Since CuZn-SOD showed major SOD activity in wild-type chloroplasts, they are expected to play a principal role for oxidative stress protection in chloroplasts.

\subsection{Effect of High-Salt on Growth and Photosynthetic Activity of csd1/2 Double-Mutant}

The protonemata of all three csd mutants ( $c s d 1, \operatorname{csd} 2$ and $\operatorname{csd1/2)}$ grew normally on agar plate under normal culture conditions. To determine whether lack of chloroplastic CuZn-SOD may reduce stress tolerance, the effects of high-salt on the growth were observed (Figure 4(a)). Under stressed conditions, csd1/2 mutant had the similar growth pattern as the wild-type. To know whether SOD isozymes other than chloroplastic CuZn-SOD may be activated in $c s d 1 / 2$ mutants grown in high-salt, SOD activities in csd1/2 mutants were analyzed (Figure 4(b)). No change in the level of activity of other SODs in the $c s d 1 / 2$ mutant was observed under high-salt conditions. These results suggest that the chloroplastic CuZn-SODs in $P$. patens do not be essential for tolerance against high-salt stress.

The maximum quantum efficiency of PSII $\left(F_{\mathrm{v}} / F_{\mathrm{m}}\right)$ in the $\operatorname{csd} 1 / 2$ mutant was similar under three different stress conditions, high-salt, high-light and heat treatments, as the wild-type (Figure 5). These results suggest that chloroplastic CuZn-SODs in $P$. patens do not contribute to tolerance against high-salt, high-light and high-temperature stress.

To examine the possibility that ROS scavenging enzymes complement the lack of chloroplastic CuZn-SODs in the double mutant, we performed RT-PCR for four ascorbate peroxidase (APX) and seven peroxiredoxin 


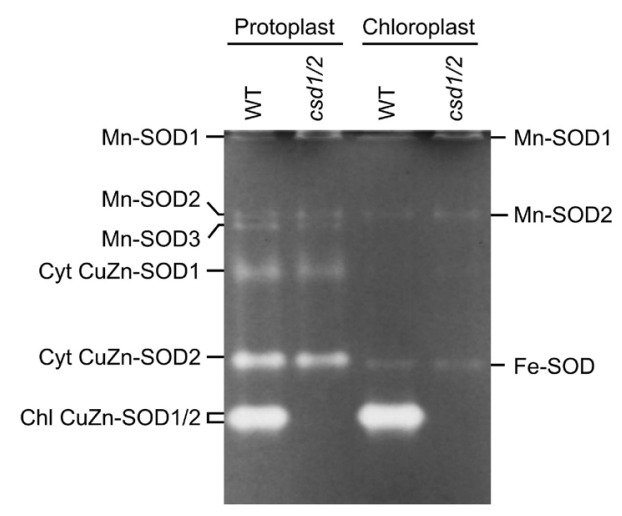

Figure 3. Identification of chloroplastic SOD isozymes in $P$. patens. Protoplasts and chloroplasts were fractionated from 6-day-old protonemata. After osmotic rupturing, extracts of protoplasts and chloroplasts ( $50 \mu \mathrm{g}$ of protein) were electrophoresed on native-PAGE gels and stained for SOD activity. Cyt, cytosolic; Chl, chloroplastic.

(a)

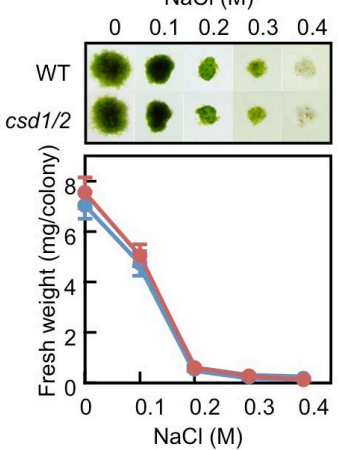

(b)

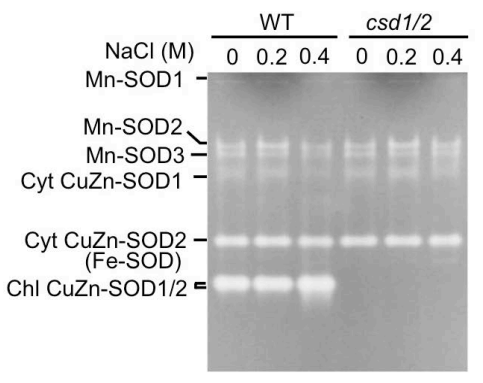

Figure 4. Comparison of high-salt tolerance in the wild-type and csd1/2 double-mutant. (a) Growth of protonemata of under high-salt conditions. Protonemata were incubated on agar plates containing BCDAT medium with increasing concentrations of $\mathrm{NaCl}$. Eight days after culture, photographs were taken (upper panel) and fresh weights of colonies were measured (lower panel). Blue and red circles indicate the WT and csd1/2, respectively. The results shown are means \pm SD of six samples; (b) SOD activity in the wild-type and double mutant. Protonemata of wild-type and csd1/2 double-mutant grown in the usual solid medium were transferred into liquid media with various concentrations of $\mathrm{NaCl}$. After incubation for $16 \mathrm{~h}$, cell free extracts were prepared. The extracts containing $60 \mu \mathrm{g}$ proteins were electrophoresed on a native polyacrylamide gel and stained for SOD activity. Cyt, cytosolic; Chl, chloroplastic.

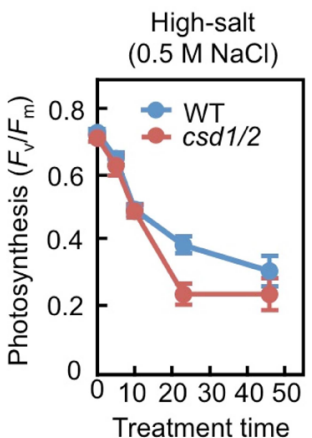

(h)
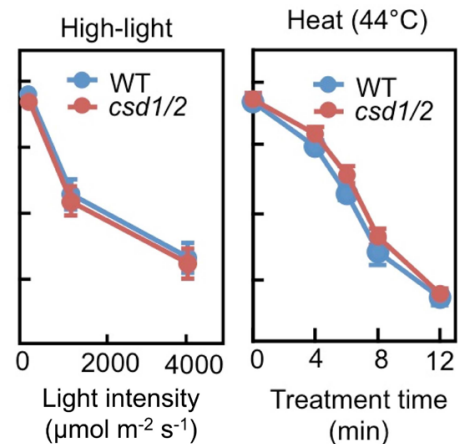

Figure 5. Comparison of photosynthetic activity in the wild-type and $c s d 1 / 2$ double-mutant after stress treatments. $F_{\mathrm{v}} / F_{\mathrm{m}}$ measurement from the wild-type and $c s d 1 / 2$ double-mutant exposed to high-salt, high-light and heat stress. Protonemata from the wild-type and the csd1/2 double-mutant were treated with liquid medium supplemented with $0.5 \mathrm{M} \mathrm{NaCl}$ (left panel) or exposed to various light intensities $\left(100-4000 \mu \mathrm{mol} \mathrm{m}{ }^{-2} \mathrm{~s}^{-1}\right)$ for $1 \mathrm{~h}$ (middle panel). For heat treatment, protonemata were treated with liquid medium at $44^{\circ} \mathrm{C}$ for various incubation time (right panel). The results shown are means $\pm \mathrm{SD}$ of four samples. 
(Prx) genes, which encode chloroplastic peroxidase. There were no differences in mRNA levels of these chloroplastic peroxidase genes between wild-type and $c s d 1 / 2$ double-mutant (Figure 6). This shows that the chloroplastic peroxidases may not complement the lack of chloroplastic CuZn-SODs in the csd1/2 double-mutant.

\subsection{Non-Photochemical Quenching (NPQ) in the Wild-Type and csd1/2 Double-Mutant}

ROS formation in chloroplasts is regulated by at least three systems, dissipation of superoxide by SOD dependent water-water cycle, appropriate supply of the electron acceptor $\left(\mathrm{NADP}^{+}\right.$) by the Calvin cycle, and dissipation of excess energy by non-photochemical quenching (NPQ) [23] [24]. Recently, Alboresi et al. [25] reported that the level and rate of NPQ induction in P. patens were higher than that in Arabidopsis after transfer to high-light conditions and speculated that the high competence of NPQ induction may predominantly contribute to high stress tolerance in the moss. In this study, lack of chloroplastic CuZn-SOD did not induce the expression of other SOD isozymes (Figure 2), but stress tolerance in chloroplasts in csd1/2 was maintained (Figure 5). Therefore, the possibility arises that a stress scavenging system other than SODs may compensate for the absence of chloroplastic CuZn-SOD. We compared NPQ under high-light conditions in the wild-type and csd1/2 double-mutant (Figure 7). The level and rate of NPQ induction in the wild-type P. patens were higher than in $A$. thaliana (Figure 7(a) and Figure 7(b)), which is consistent with the result by Alboresi et al. [25]. NPQ induction in the csd1/2 double-mutant was the similar as observed in the wild-type, suggesting that the NPQ regulation system was not affected by a lack of chloroplast CuZn-SOD. Alboresi et al. [25] speculated that NPQ regulation is the major system for the suppression of high-light induced ROS in chloroplasts of $P$. patens. However, the contribution of SOD system remains unknown. Therefore, we examined this question using NPQ inhibitors (Figure 7(c) and Figure 7(d)). NPQ is mainly associated with the xanthophyll cycle and transthylakoid proton gradient $(\Delta \mathrm{pH})$. We analyzed the effect of DTT (xanthophyll cycle inhibitor) and nigericin (uncoupler) on NPQ in the wild-type protonemata (Figure 7(c)). DTT caused partial inhibition of NPQ, while nigericin caused compete inhibition of NPQ, suggesting that $\Delta \mathrm{pH}$ is essential for NPQ in P. patens protonemata. To evaluate chloroplastic CuZn-SOD and NPQ contribution to the suppression of high-light induced ROS stress, effects of uncoupler on $F_{\mathrm{v}} / F_{\mathrm{m}}$ was compared between the wild-type and $\operatorname{csd} 1 / 2$ mutant (Figure 7(d)). Nigericin promoted the inhibition of $F_{\mathrm{v}} / F_{\mathrm{m}}$ under high-light conditions in both the wild-type and the $c s d 1 / 2$ double-mutant, but there was no difference in the degree of inhibition between the wild-type and the csd1/2 double-mutant. This suggests that NPQ was the major component in the regulation of ROS suppression, while chloroplastic CuZn-SODs do not contribute to it.

\section{Discussion and Conclusions}

The most prominent feature in $P$. patens $c s d 1 / 2$ mutant is the fact that the mutant chloroplasts maintained

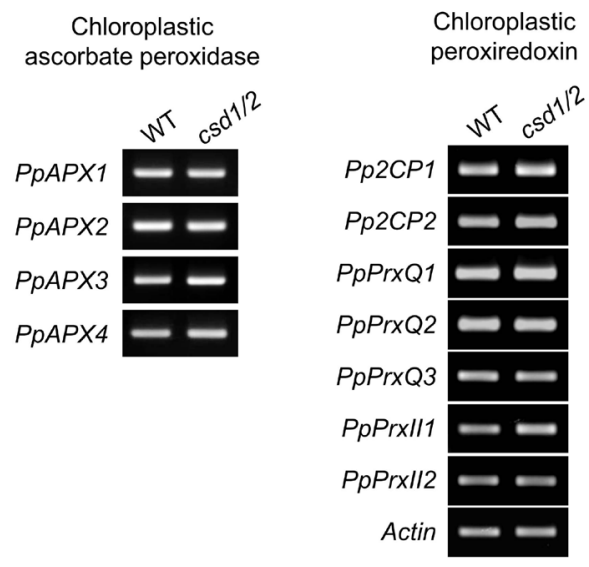

Figure 6. Expression levels of chloroplastic peroxidase genes. Total RNA was extracted from $P$. patens protonemata cultured on BCDAT solid medium for $6 \mathrm{~d}$ and subjected to RT-PCR using primers for chloroplastic ascorbate peroxidase genes (PpAPX1, PpAPX2, PpAPX3, PpAPX4) and peroxiredoxin genes: 2-cysteine peroxiredoxin (Pp2CP1, Pp2CP2), peroxiredoxin Q (PpPrxQ1, PpPrxQ2, PpPrxQ3), and peroxiredoxin II (PpPrxII1, PpPrxII2). 

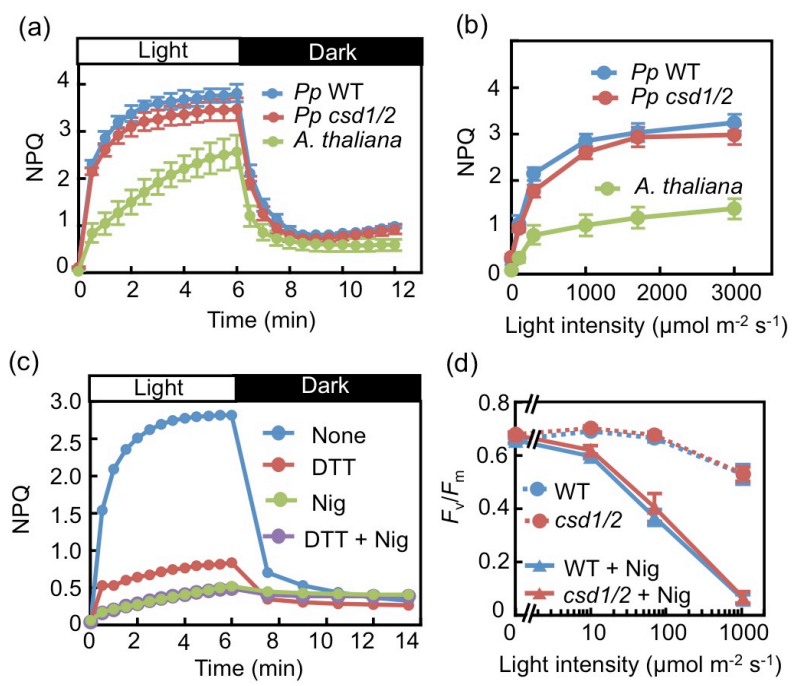

(d)

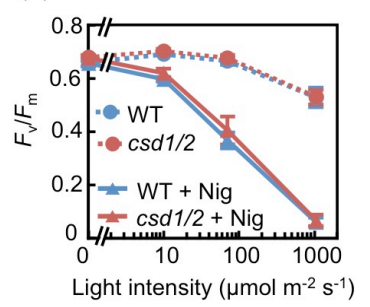

Figure 7. NPQ in wild-type and csd1/2 double-mutant protonemata. (a) Comparison of NPQ induction in the P. patens (Pp) wild-type, csd1/2 double-mutant and A. thaliana. NPQ was measured for $6 \mathrm{~min}$ under $1000 \mu \mathrm{mol} \mathrm{m}^{-2} \mathrm{~s}^{-1}$ actinic light (Light). After measurement, the light was turned off and NPQ was measured for 6 min (Dark); (b) NPQ of the P. patens (Pp) wild-type, csd1/2 double-mutant and $A$. thaliana under various light conditions. NPQ was measured at 1-min exposure to actinic light $\left(0-3000 \mu \mathrm{mol} \mathrm{m} \mathrm{m}^{-2}\right)$; (c) Effect of NPQ inhibitors on NPQ induction in wild-type protonemata. The wild-type protonemata were pre-incubated in liquid BCDAT media with $1 \mathrm{mM}$ DTT or/and $10 \mu \mathrm{M}$ nigericin (Nig) or without inhibitor (None) for $30 \mathrm{~min}$ in the dark. NPQ was measured under $1000 \mu \mathrm{mol} \mathrm{m}^{-2} \mathrm{~s}^{-1}$ actinic light for $6 \mathrm{~min}$ (Light), following an 8-min dark incubation (Dark); (d) Effect of high-light on $F_{\mathrm{v}} / F_{\mathrm{m}}$ in the wild-type and csd1/2 double-mutant after $10 \mu \mathrm{M}$ nigericin treatment. The wild-type and $c s d 1 / 2$ protonemata were pre-incubated in liquid BCDAT media with $10 \mu \mathrm{M}$ nigericin (Nig) or without inhibitor for $30 \mathrm{~min}$ in the dark. Subsequently, the protonemata were exposed to various light intensities (0 $1000 \mu \mathrm{mol} \mathrm{m} \mathrm{s}^{-1}$ ) for $1 \mathrm{~h}$, and then measured $F_{\mathrm{v}} / F_{\mathrm{m}}$.

high-salt, high-light, and high-temperature stress tolerance despite the complete loss of the major chloroplast CuZn-SOD (Figure 4 and Figure 5), which is a key enzyme in the water-water cycle [14]. However, the contribution of CuZn-SOD to ROS suppression in chloroplasts can be complemented by the co-existence of Fe-SOD [13]. Under copper-deficient conditions, decreased CuZn-SOD activity and increased Fe-SOD were reported in the moss Barbula unguiculata [9] [10] and seed plants [1] [11] [12]. In contrast, the level of mRNA and the catalytic activity of SOD isozymes, including Fe-SOD, were not changed in P. patens by the complete loss of chloroplastic CuZn-SOD (Figure 2 and Figure 3). Therefore, the cause of sustained stress tolerance in the $\operatorname{csd} 1 / 2$ mutant was not explained by the complementation due to the increased expression of other SODs.

The minor contribution of chloroplastic CuZn-SOD to stress tolerance in the chloroplasts of $P$. patens may be the reason why chloroplastic CuZn-SOD genes were preferentially suppressed by copper deficiency [8]. The preferential repression of chloroplastic CuZn-SOD in copper-deficient $P$. patens is unique. Since copper is the prosthetic metal in CuZn-SOD, CuZn-SOD inactivation is inevitable under conditions of copper deficiency [13]. In $P$. patens, however, cytosolic CuZn-SOD activity was maintained after repeated subcultures in copper deficient medium [8]. Further, copper deficiency induces transcriptional repression of both chloroplastic and cytosolic CuZn-SOD via miR398 in seed plants, including Arabidopsis [26]. P. patens lacks miR398, but has miR1073, which is involved in the preferential degradation of chloroplastic CuZn-SOD mRNA [8]. Preferential degradation of chloroplastic CuZn-SOD mRNA and robust maintenance of cytosolic CuZn-SOD activity suggest that SODs other than CuZn-SOD have a principal role in stress tolerance in chloroplasts.

The reduced SOD activity in chloroplasts but normal growth observed in $P$. patens has previously been reported in the Arabidopsis CCS (copper chaperone for CuZn-SOD) mutant [27]. Although the CCS mutant had largely impaired CuZn-SOD activity, it maintained normal regulation of Fe-SOD expression by copper availability. Therefore, when the CCS mutant was grown in copper-sufficient medium, it showed no measurable SOD activity in chloroplasts and no photosynthetic deficiencies, suggesting that trace amounts of CuZn-SOD or Fe-SOD in chloroplasts could maintain normal chloroplast function [27]. Fe-SOD knockout mutants showed that Fe-SODs are essential for chloroplast development in Arabidopsis [28]. Therefore, it is possible that minor 
Fe-SOD and/or Mn-SOD isozymes in chloroplasts are essential for normal chloroplast function in P. patens. The presence of Mn-SOD in the chloroplasts has yet to be examined because the amino acid sequences of the N-terminal region of the three PpMSD1 variants are identical and predicted to be localized to mitochondria. Therefore, further analysis is required to identify the chloroplast-targeting transcript variant.

Compared with Arabidopsis, P. patens exhibits a higher level of and more rapid NPQ induction during the transfer to high-light conditions [25]. We observed this property in both the wild type and the csd1/2 mutant (Figure 7). The inhibition of NPQ by nigericin promoted the inhibition of photosynthetic activity (Figure 7(d)), suggesting that NPQ plays a major protective role in chloroplasts under high-light stress. Because the degree of inhibition was identical in the wild-type moss and $c s d 1 / 2$ mutant, it is possible that chloroplastic CuZn-SODs do not contribute to the NPQ-dependent protection of chloroplasts against high-light stress. However, it does not mean that SODs other than CuZn-SOD do not contribute to stress tolerance in chloroplasts. In the future, the contribution of the chloroplast-localized minor Mn-SOD and Fe-SOD to stress tolerance will be evaluated when the effect of high-light treatment on a mutant lacking chloroplastic Mn-SOD and Fe-SOD is measured under NPQ-suppressed conditions.

\section{Acknowledgements}

This work was supported by Grants-in-Aid for Scientific Research (C) (No. 22570046 to H.T. and K.T. and 22580203 to S.T.) and for Young Scientists (B) (No. 22770044 to K.T.) from the Japan Society for the Promotion of Science, and a grant from the Toray Science Foundation to H.T. and K.T.

\section{References}

[1] Bowler, C., Van Montagu, M. and Inzé, D. (1992) Superoxide Dismutase and Stress Tolerance. Annual Review of Plant Physiology and Plant Molecular Biology, 43, 83-116. http://dx.doi.org/10.1146/annurev.pp.43.060192.000503

[2] Bowler, C., Van Camp, W., Van Montagu, M., Inzé, D. and Asada, K. (1994) Superoxide Dismutase in Plants. Critical Reviews in Plant Sciences, 13, 199-218. http://dx.doi.org/10.1080/07352689409701914

[3] Takahashi, M.A. and Asada, K. (1983) Superoxide Anion Permeability of Phospholipid Membranes and Chloroplast Thylakoids. Archives of Biochemistry and Biophysics, 226, 558-566. http://dx.doi.org/10.1016/0003-9861(83)90325-9

[4] Mehler, A.H. (1951) Studies on Reactions of Illuminated Chloroplasts. II. Stimulation and Inhibition of the Reaction with Molecular Oxygen. Archives of Biochemistry and Biophysics, 34, 339-351. http://dx.doi.org/10.1016/0003-9861(51)90012-4

[5] Schaefer, D.G. and Zrÿd, J.P. (1997) Efficient Gene Targeting in the Moss Physcomitrella patens. The Plant Journal, 11, 1195-1206. http://dx.doi.org/10.1046/j.1365-313X.1997.11061195.X

[6] Rensing, S.A., Lang, D., Zimmer, A.D., Terry, A., Salamov, A., Shapiro, H., Nishiyama, T., Perroud, P.F., Lindquist, E.A., Kamisugi, Y., Tanahashi, T., Sakakibara, K., Fujita, T., Oishi, K., Shin-I, T., Kuroki, Y., Toyoda, A., Suzuki, Y., Hashimoto, S., Yamaguchi, K., Sugano, S., Kohara, Y., Fujiyama, A., Anterola, A., Aoki, S., Ashton, N., Barbazuk, W.B., Barker, E., Bennetzen, J.L., Blankenship, R., Cho, S.H., Dutcher, S.K., Estelle, M., Fawcett, J.A., Gundlach, H., Hanada, K., Heyl, A., Hicks, K.A., Hughes, J., Lohr, M., Mayer, K., Melkozernov, A., Murata, T., Nelson, D.R., Pils, B., Prigge, M., Reiss, B., Renner, T., Rombauts, S., Rushton, P.J., Sanderfoot, A., Schween, G., Shiu, S.H., Stueber, K., Theodoulou, F.L., Tu, H., Van de Peer, Y., Verrier, P.J., Waters, E., Wood, A., Yang, L., Cove, D., Cuming, A.C., Hasebe, M., Lucas, S., Mishler, B.D., Reski, R., Grigoriev, I.V., Quatrano, R.S. and Boore, J.L. (2008) The Physcomitrella Genome Reveals Evolutionary Insights into the Conquest of Land by Plants. Science, 319, 64-69. http://dx.doi.org/10.1126/science.1150646

[7] Frank, W., Ratnadewi, D. and Reski, R. (2005) Physcomitrella patens Is Highly Tolerant against Drought, Salt and Osmotic Stress. Planta, 220, 384-394. http://dx.doi.org/10.1007/s00425-004-1351-1

[8] Higashi, Y., Takechi, K., Takano, H. and Takio, S. (2013) Involvement of MicroRNA in Copper Deficiency-Induced Repression of Chloroplastic CuZn-Superoxide Dismutase Genes in the Moss Physcomitrella patens. Plant \& Cell Physiology, 54, 1345-1355. http://dx.doi.org/10.1093/pcp/pct084

[9] Shiono, T., Nakata, M., Yamahara, T., Matuzaki, M., Deguchi, H. and Satoh, T. (2003) Repression by Cu of the Expression of Fe-Superoxide Dismutase of Chloroplasts in the Moss Barbula unguiculata But Not in the Liverwort Marchantia paleacea var. diptera. Journal of the Hattori Botanical Laboratory, 93, 141-153.

[10] Nagae, M., Nakata, M. and Takahashi, Y. (2008) Identification of Negative cis-Acting Elements in Response to Copper in the Chloroplastic Iron Superoxide Dismutase Gene of the Moss Barbula unguiculata. Plant Physiolology, 146, 1687-1696. http://dx.doi.org/10.1104/pp.107.114868 
[11] Abdel-Ghany, S.E., Müller-Moulé, P., Niyogi, K.K., Pilon, M. and Shikanai, T. (2005) Two P-Type ATPases Are Required for Copper Delivery in Arabidopsis thaliana Chloroplasts. Plant Cell, 17, 1233-1251. http://dx.doi.org/10.1105/tpc.104.030452

[12] Kurepa, J., Van Montagu, M. and Inzé, D. (1997) Expression of sodCp and sodB Genes in Nicotiana tabacum: Effects of Light and Copper Excess. Journal of Experimental Botany, 48, 2007-2014. http://dx.doi.org/10.1093/jxb/48.12.2007

[13] Pilon, M., Ravet, K. and Tapken, W. (2011) The Biogenesis and Physiological Function of Chloroplast Superoxide Dismutases. Biochimica et Biophysica Acta, 1807, 989-998. http://dx.doi.org/10.1016/j.bbabio.2010.11.002

[14] Asada, K. (2000) The Water-Water Cycle as Alternative Photon and Electron Sinks. Philosophical Transactions of the Royal Society, 355, 1419-1431. http://dx.doi.org/10.1098/rstb.2000.0703

[15] Nishiyama, T., Hiwatashi, Y., Sakakibara, K., Kato, M. and Hasebe, M. (2000) Tagged Mutagenesis and Gene-Trap in the Moss, Physcomitrella patens by Shuttle Mutagenesis. DNA Research, 7, 9-17. http://dx.doi.org/10.1093/dnares/7.1.9

[16] Hofmann, N.R. and Theg, S.M. (2003) Physcomitrella patens as a Model for the Study of Chloroplast Protein Transport: Conserved Machineries between Vascular and Non-Vascular Plants. Plant Molecular Biology, 53, 643-654. http://dx.doi.org/10.1023/B:PLAN.0000019065.31490.06

[17] Bradford, M.M. (1976) A Rapid and Sensitive Method for the Quantitation of Microgram Quantities of Protein Utilizing the Principal of Protein Dye Binding. Analytical Biochemistry, 72, 248-254. http://dx.doi.org/10.1016/0003-2697(76)90527-3

[18] Laemmli, U.K. (1970) Cleavage of Structural Proteins during the Assembly of the Head of Bacteriophage T4. Nature, 227, 680-685. http://dx.doi.org/10.1038/227680a0

[19] Beyer, W.F. and Fridovich, I. (1987) Assaying for Superoxide Dismutase Activity: Some Large Consequence of Minor Change in Conditions. Analytical Biochemistry, 161, 559-566. http://dx.doi.org/10.1016/0003-2697(87)90489-1

[20] Murray, M.G. and Thompson, W.F. (1980) Rapid Isolation of High Molecular Weight Plant DNA. Nucleic Acids Research, 8, 4321-4326. http://dx.doi.org/10.1093/nar/8.19.4321

[21] Suzuki, T., Takio, S. and Satoh, T. (1998) Light-Dependent Expression in Liverwort Cells of chlL/N and chlB Identified as Chloroplast Genes Involved in Chlorophyll Synthesis in the Dark. Journal of Plant Physiology, 152, 31-37. http://dx.doi.org/10.1016/S0176-1617(98)80098-9

[22] Hernández, J.A., Campillo, A., Jiménez, A., Alarcón, J.J. and Sevilla, F. (1999) Response of Antioxidant Systems and Leaf Water Relations to NaCl Stress in Pea Plants. New Phytologist, 141, 241-251. http://dx.doi.org/10.1046/j.1469-8137.1999.00341.x

[23] Ort, D.R. and Baker, N.R. (2002) A Photoprotective Role for $\mathrm{O}_{2}$ as an Alternative Electron Sink in Photosynthesis? Current Opinion in Plant Biology, 5, 193-198. http://dx.doi.org/10.1016/S1369-5266(02)00259-5

[24] Proctor, M.C. and Smirnoff, N. (2011) Ecophysiology of Photosynthesis in Bryophytes: Major Roles for Oxygen Photoreduction and Non-Photochemical Quenching? Physiologia Plantarum, 141, 130-140. http://dx.doi.org/10.1111/j.1399-3054.2010.01424.x

[25] Alboresi, A., Gerotto, C., Giacometti, G.M., Bassi, R. and Morosinotto, T. (2010) Physcomitrella patens Mutants Affected on Heat Dissipation Clarify the Evolution of Photoprotection Mechanisms upon Land Colonization. Proceedings of the National Academy of Science of the United States of America, 107, 11128-11133. http://dx.doi.org/10.1073/pnas.1002873107

[26] Sunkar, R., Kapoor, A. and Zhu, J.K. (2006) Posttranscriptional Induction of Two Cu/Zn Superoxide Dismutase Genes in Arabidopsis Is Mediated by Downregulation of miR398 and Important for Oxidative Stress Tolerance. Plant Cell, 18, 2051-2065. http://dx.doi.org/10.1105/tpc.106.041673

[27] Cohu, C.M., Abdel-Ghany, S.E., Gogolin Reynolds, K.A., Onofrio, A.M., Bodecker, J.R., Kimbrel, J.A., Niyogi, K.K. and Pilon, M. (2009) Copper Delivery by the Copper Chaperone for Chloroplast and Cytosolic Copper/Zinc-Superoxide Dismutases: Regulation and Unexpected Phenotypes in an Arabidopsis Mutant. Molecular Plant, 2, 1336-1350. http://dx.doi.org/10.1093/mp/ssp084

[28] Myouga, F., Hosoda, C., Umezawa, T., Iizumi, H., Kuromori, T., Motohashi, R., Shono, Y., Nagata, N., Ikeuchi, M. and Shinozaki, K. (2008) A Heterocomplex of Iron Superoxide Dismutases Defends Chloroplast Nucleoids against Oxidative Stress and Is Essential for Chloroplast Development in Arabidopsis. Plant Cell, 20, 3148-3162.

http://dx.doi.org/10.1105/tpc.108.061341 